This is a peer-reviewed, accepted author manuscript of the following article: Verzobio, A., El-Awady, A., Ponnambalam, K., Quigley, J., \& Zonta, D. (Accepted/In press). An elicitation process to quantify Bayesian networks for dam failure analysis. Canadian Journal of Civil Engineering.

\title{
1 An elicitation process to quantify Bayesian networks for dam failure analysis
}

2 Andrea Verzobio ${ }^{\mathrm{a},{ }^{*}}$, Ahmed El-Awady ${ }^{\mathrm{b}, \mathrm{c}}$, Kumaraswamy Ponnambalam ${ }^{\mathrm{c}}$, John Quigley ${ }^{\mathrm{d}}$ and

3 Daniele Zonta ${ }^{\mathrm{e}}$

$4 \quad{ }^{a}$ Department of Civil and Environmental Engineering, University of Strathclyde, Glasgow, UK;

5 andrea.verzobio@strath.ac.uk

$6 \quad{ }^{b}$ Department of Environmental Engineering Science, Institute of Environmental Studies and

7 Research, Ain Shams University, Cairo, Egypt; ahmed.awady@iesr.asu.edu.eg

$8{ }^{\mathrm{c}}$ Department of Systems Design Engineering, University of Waterloo, Ontario, Canada;

9 aelawady@uwaterloo.ca,ponnu@uwaterloo.ca

10 dDeptartment of Management Science, University of Strathclyde, Glasgow, UK;

11 j.quigley@strath.ac.uk

12 e Department of Civil, Environmental and Mechanical Engineering, University of Trento, Trento,

13 Italy; daniele.zonta@unitn.it

$14{ }^{*}$ Corresponding author: Andrea Verzobio, Department of Civil and Environmental Engineering,

15 University of Strathclyde, 75 Montrose Street, Glasgow, G1 1XJ, Scotland, UK; 07842880677;

16 andrea.verzobio@strath.ac.uk

17 Word count: 9506 


\section{Abstract}

Bayesian Networks support the probabilistic failure analysis of complex systems, e.g. dams and

20 bridges, needed for a better understanding of the system reliability and for taking mitigation

21 actions. In particular, they are useful in representing graphically the interactions among system

22 components, while the quantitative strength of the interrelationships between the variables is

23 measured using conditional probabilities. However, due to a lack of objective data it often becomes

24 necessary to rely on expert judgment to provide subjective probabilities to quantify the model.

25 This paper proposes an elicitation process that can be used to support the collection of valid and

26 reliable data with the specific aim of quantifying a Bayesian Network, while minimizing the

27 adverse impact of biases to which judgment is commonly subjected. To illustrate how this

28 framework works, it is applied to a real-life case study regarding the safety of the Mountain Chute

29 Dam and Generating Station, which is located on the Madawaska River in Ontario, Canada. This

30 contribution provides a demonstration of the usefulness of eliciting engineering expertise with

31 regard to system reliability analysis.

\section{Keywords}

33 Dam safety, Bayesian network, statistical inference, elicitation, expert knowledge, expert 34 judgment.

\section{1. Introduction}

Dams fail due to a combination of more frequent load and reduced resistance to the load

37 exceeding the facility's capacity, design problems, unexpected flood events or inappropriate

38 decisions in managing dams. Such failures, including breaches, may lead to catastrophic events

39 which affect both properties and lives of people. Maintaining dams is challenging, as resources 
such as labor and capital are limited, facilities are remote and usage profiles are uncertain. Global

41 weather patterns have been changing, causing periods of flooding, which have resulted in an

42 increase in operating the dams. Understanding and anticipating the environment in which the dams

43 will operate is vital for maintaining the availability of the asset. Effectively maintaining the asset

44 requires a mathematical model to explicate the relationship between environment, usage, hazards

45 and management decisions, and to support the optimal long-term productivity of the asset.

46 While several examples of mathematical and probabilistic approaches used to evaluate the

47 safety of dams can be found in the literature (Yanmaz \& Gunindi, 2008) (Li, et al., 2011)

48 (Goodarzi, et al., 2012) (Su, et al., 2015), in this contribution we decide to use the Bayesian

49 Network $(\mathrm{BN})$ since it has many advantages and it is an increasingly popular method for reasoning

50 under uncertainty and modelling uncertain domains. For instance, in comparison with two most

51 commonly used approaches, i.e. the Event Tree Analysis (ETA) and the Fault Tree Analysis

52 (FTA), BNs can more succinctly represent the dependency relationship between a large number of

53 variables, permit variables to be described in multiple states not just binary, i.e. true or false,

54 describe and represent multiple initiating events, and explicitly integrate different types of data,

55 e.g. technical, environmental and social, in a single unified representation. Comparisons between

56 BN and ETA or FTA in safety analysis can be found in (Khakzad, et al., 2011) (Jong \& Leu, 2013)

57 (Zerrouki \& Tamrabet, 2015a) (Zerrouki \& Tamrabet, 2015b).

58 BNs provide a powerful framework for reasoning under uncertainty, and consequently have

59 been recently applied to various engineering problems, e.g. earthquake risk management

60 (Bayraktarli, et al., 2005) (Bensi, et al., 2011) (Liu \& Nadim, 2013), avalanche risk assessment

61 (Gret-Regamey \& Straub, 2006), landslide hazard mitigation (Medina-Cetina \& Nadim, 2008),

62 reliability analysis (Langsetha \& Portinaleb, 2007), climate change assessment (Peter, et al., 2009),

63 risk assessment in maritime engineering (Kelangath, et al., 2011), environmental modelling and 
management (Aguilera, et al., 2011), risk assessment for fatigue damage (Sankararaman, et al.,

65 2011) (Ling \& Mahadevan, 2012), scour management (Maroni, et al., 2019). In addition, as regards

66 the topic of this paper, in the literature we can find many papers in which BNs are used to develop

67 dam safety analysis, among the many we recommend (Smith, 2006) (Xu, et al., 2011) (Zhang, et

68 al., 2011) (Miroslaw-Swiatek, et al., 2012) (Peng \& Zhang, 2013) (Ahmadi, et al., 2015) (Gang,

69 et al., 2016) (Eldosouky, et al., 2017) (Liu, et al., 2017) (Briseno-Ramiro, et al., 2019)

70 (Dassanavake \& Mousa, 2020).

71 Specifically, BNs are probabilistic graphical models that use directed acyclic graph to represent

72 a set of uncertain variables and their conditional dependencies (Charniak, 1991) (Ben Gal, 2007)

73 (Jensen \& Nielsen, 2007). In detail, nodes represent the collection of random variables, while

74 edges represent the interrelationship between these variables. While the topology of the BN

75 provides the causal structuring of the problem under study, the quantitative strength of the

76 interrelationships among variables is measured using conditional probability distributions, which

77 can be updated when new data become available. Typically, the quantification of the probabilities

78 may be obtained from statistical and historical data, existing physical or empirical models and

79 logic inference. However, these quantification sources and methodologies are often not easy to be

80 conducted and not sufficient to quantify the entire BN, due to the lack of sufficient models that

81 interpret the interrelationships among system variables and due to the lack of data and information.

82 Consequently, it becomes necessary to rely on expert judgments to quantify these dependencies:

83 engineering knowledge and experience can be an important data source for estimating these

84 probabilities (Dias, et al., 2018).

85 Eliciting expert judgment in the form of subjective probabilities is a socio-technical activity.

86 As such it requires a structured and facilitated process to extract meaningful judgments because

87 people, even experts, are unable to provide accurate and reliable data simply on request (Ferrell, 
1994) (Vick, 2002). An example about discrepancies between experts in risk assessment can be

89 found in (Rizak \& Hrudey, 2005). In addition, since the work of Tversky and Kahneman in the 90 early 1970s (Tversky \& Kahneman, 1974), there has been awareness of the biases and heuristics

91 people apply in decision-making under uncertainty that can result in poor probability assessments.

92 Elicitation processes are designed to minimize the influence of these biases (Quigley \& Walls,

93 2020). In the literature, there are a variety of existing processes for eliciting expert knowledge with

94 engineering applications, see for instance (Bubniz, et al., 1998), (Hodge, et al., 2001) and

95 (Astfalck, et al., 2018). Textbooks such as (Cooke, 1991), (Meyer \& Booker, 1991) and (Dias, et

96 al., 2018) are references for general aspects of elicitation. However, very little has been reported

97 about elicitation processes aimed specifically at quantifying BNs using expert judgment

98 (Sigurdsson, et al., 2001) (Norrington, et al., 2008) (Christophersen, et al., 2018), especially for

99 civil engineering applications, where we require experts to assess a variety of dependent variables,

100 each of which is in one of several possible states. In particular, a methodology to support the

101 collection of valid and reliable data in order to quantify the BN is not available.

102 In this paper, the aim is to develop a methodology for eliciting expert knowledge in the specific

103 case where the model is described by a BN. We start with an introduction of the fundamentals of

104 BNs in section 2. In section 3, a four-stage structured elicitation process is developed generically

105 so that it can be applied to many civil engineering structures, e.g. dams and bridges. Section 4

106 presents an implementation of this methodology, with its application to a real-life case study

107 regarding the safety of the Mountain Chute Dam and Generating Station, which is situated on the

108 Madawaska River in Ontario, Canada. Concluding remarks, along with the explanation of the

109 lessons learnt from the application, are presented at the end of the paper. 


\section{Bayesian Networks}

111 Bayesian Networks (BNs), also known as Bayes networks, belief networks or decision

112 networks, are probabilistic graphical models used to represent knowledge about an uncertain

113 domain using a combination of principles from graph theory, probability theory, computer science,

114 and statistics (Charniak, 1991) (Ben Gal, 2007) (Jensen \& Nielsen, 2007). In the graph, nodes

115 represent the collection of random variables, while edges represent the interrelationship between

116 these variables. In addition, each node is associated with conditional probability values that model

117 the uncertain relationship between the node and its parents; they compose the so-called node

118 probability table (NPT). BNs can model the quantitative strength of the interrelationships among

119 variables, i.e. the nodes, allowing their probabilities to be updated using any new available data

120 and information. They are mathematically rigorous, understandable, and efficient in computing

121 joint probability distribution over a set of random variables, and consequently very useful in

122 supporting risk analysis of complex systems.

123 BNs are probabilistic graphical models that use directed acyclic graph (DAG): this means that 124 a set of directed edges are used to connect the set of nodes, where these edges represent direct

125 statistical dependencies among variables, with the constraint of not having any directed cycles. Let

$126 \mathrm{X}=\left(\mathrm{X}_{1}, \ldots, \mathrm{X}_{\mathrm{i}}, \ldots, \mathrm{X}_{\mathrm{n}}\right)$ represent the set of nodes, i.e. the uncertain variables. A node $\mathrm{X}_{\mathrm{j}}$ is called

127 parent of a child node $X_{i}$ if there is a directed edge from node $X_{j}$ to node $X_{i}$, meaning that $X_{i}$

128 depends on $\mathrm{X}_{\mathrm{j}}$. Each node can have many parents nodes, while nodes with no parent are called root

129 nodes and nodes with no child are called leaf nodes. In addition, each root node is associated with

130 a basic probability table (BPT), while each child node with a conditional probability table (CPT).

131 The joint probability function of random variables in a $\mathrm{BN}$ can be expressed as follows: 


$$
P(X)=\prod_{i=1}^{n} P\left[X_{i} \mid P a\left(X_{i}\right)\right]
$$

133 where $\mathrm{P}(\mathrm{X})$ is the joint probability and $\mathrm{Pa}\left(\mathrm{X}_{\mathrm{i}}\right)$ is the parent set of node $\mathrm{X}_{\mathrm{i}}$. If $\mathrm{X}_{\mathrm{i}}$ has no parents, i.e.

134 it is a root node, then the function reduces to the unconditional probability of $\mathrm{P}\left(\mathrm{X}_{\mathrm{i}}\right)$. A simple

135 example of $\mathrm{BN}$ with three variables as regards dam safety analysis is shown in Figure 1: both the

136 severity of the flood and a high-water pressure can cause the presence of seepage in the dam; in

137 addition, the flood severity has a direct effect on the level of water pressure. The table related to

138 the flood severity, that is a root node, represent an example of BPT, while the tables of the other

139 two child nodes are examples of CPT.

140 Figure 1. An example of BN with three variables.

\begin{tabular}{|c|c|c|}
\hline & \multicolumn{2}{|c|}{ WP } \\
\hline FS & Low & High \\
\hline Low & 0.9 & 0.1 \\
\hline High & 0.6 & 0.4 \\
\hline
\end{tabular}
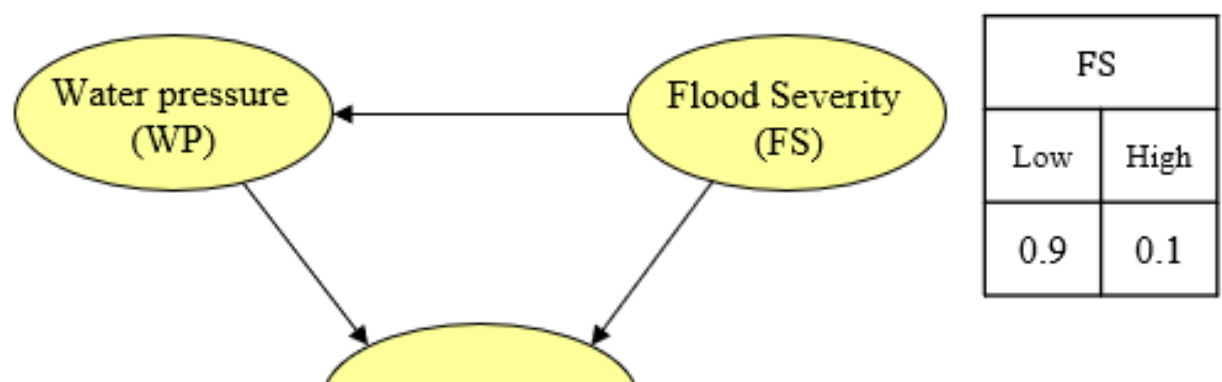

Seepage (SE)

\begin{tabular}{|c|c|c|c|}
\hline & & \multicolumn{2}{|c|}{$\mathrm{SE}$} \\
\hline WP & $\mathrm{FS}$ & Yes & No \\
\hline Low & Low & 0.1 & 0.9 \\
\hline High & Low & 0.5 & 0.5 \\
\hline Low & High & 0.3 & 0.7 \\
\hline High & High & 0.7 & 0.3 \\
\hline
\end{tabular}

142 Generically, in BNs there are two main types of reasoning: predictive reasoning, i.e. top-down 
143 or forward reasoning, in which evidence nodes are connected through parent nodes (cause to

144 effect), and diagnostic reasoning, i.e. bottom-up or backward reasoning, in which evidence nodes 145 are connected through child nodes (effect to cause).

146 Finally, we can summarize how to build and use a BN with three steps: structuring the problem, 147 defining the conditional probabilities, and making the final inference. The first step aims to define

148 the topology of the BN: first, the relevant variables of the problem are identified and expressed as

149 statistical variables, discrete or continuous; then, the network is created by joining the variables

150 according to their dependency. The second step is about quantifying the interrelationship among

151 connected nodes, i.e. defining the CPTs, as well as the BPTs in the case of root nodes. They may

152 be obtained from statistical and historical data, existing physical or empirical models, logic

153 inference or they may be elicited from experts. Lastly, the inference step concerns entering the

154 evidence in the $\mathrm{BN}$, updating the probabilities, and interpreting the final results.

\section{3. Elicitation Process for Bayesian Networks}

156 In this paper, the aim is to support the collection of valid and reliable data in order to quantify 157 a BN, by developing a methodology for the specific case where the topology of the BN has already

158 been defined, i.e. with the problem already structured. In this case, the elicitation process is then 159 required to extract and quantify the subjective judgments about the uncertain quantities, which are 160 the conditional probabilities that represent the interrelationships among connected nodes.

161 There are various protocols for probability elicitation (Morgan, et al., 1990), for a recent review 162 see (Quigley \& Walls, 2020). The methodology proposed in this contribution is adapted from the 163 Stanford Research Institute (SRI) model (Ferrell, 1985) (Spetzler \& Stael Von Holstein, 1985) 164 (Merkhofer, 1987). Accordingly, the process for eliciting expert judgment is based on seven 165 possible stages: motivating the experts with the aims of the elicitation process, structuring the 
uncertain quantities in an unambiguous way, conditioning the expert's judgement to avoid

167 cognitive biases, encoding the probability distributions, verifying the consistency of the elicited 168 distributions, aggregating probabilities from different experts and discretizing continuous

169 probability distributions. Moreover, to conduct an elicitation process at least two characters are

170 necessary: a subject, i.e. the expert, and an analyst, i.e. the interviewer. The first one provides

171 expertise, i.e. he/she is "a person with substantive knowledge about the events whose uncertainty

172 is to be assessed" (Ferrell, 1985), while the second one has responsibility for designing, developing

173 and executing the process as well as evaluating the procedures. For the rule of analyst, also called

174 facilitator, it is common to have at least one person who is very knowledgeable in elicitation 175 practice and can manage the process, and another one with wide expertise in the area of the design 176 project.

177 Starting from the SRI protocol and according to the specific requirements of a BN, we develop 178 a four-stage structured methodology to support the elicitation meaningfully. In the next subsection 179 each stage is extensively presented by defining each phase of the process, presenting the roles of 180 the key personnel and highlighting all the potential biases that may influence the process, while 181 proposing appropriate actions in order to minimize the risk of a biased judgment.

\section{$182 \quad 3.1 \quad$ The four-stage structured elicitation process}

183 In the following, each stage of the process is presented in detail; the flowchart in Figure 2 shows

184 the proposed elicitation process.

185 Stage 1: Selecting. To start, the analysts have to study carefully the project and the proposed

$186 \mathrm{BN}$, to understand which kind of expertise is required: it is fundamental to ensure coverage of all

187 the different aspects of the problem, so more than one expert is usually necessary. This is even 188 more important in civil engineering applications, because in this field experts are usually very 
190 experts and build up profiles of experts who may be able to answer questions concerning the 191 quantities of interest, i.e. the values required to be quantified in the BN. Constructing a profile 192 matrix can be useful (Bolger, 2018), which matches the knowledge requirements with the expert 193 roles: it supports the identification of expertise needed as well as justification for the choice of 194 experts. The number of required experts depends then on the variability of expertise per domain. 195 Adding as many experts as possible seems beneficial, however, practically it may be difficult to 196 manage many experts and there will be a diminishing return on adding more experts. In addition, 197 we have to be aware that in real-world it is not so easy to have the availability of many experts. 198 Once the experts have been selected, the analysts have to arrange meetings to conduct interviews. 199 Prior to the meetings, it is recommended to give to the experts an outline about the project and 200 where their knowledge will be useful, so that they have the opportunity to reflect upon the events. 201 Stage 2: Structuring. Individual interviews between the analysts and the selected experts are 202 conducted. The initial part of the interview has two purposes: to introduce the expert to the 203 encoding task as well as identifying and addressing motivational biases (Fischhoff, 1989), such as 204 management bias and expert bias. Management bias occurs when experts provide goals rather than 205 judgments, e.g. "the dam will not fail", while expert bias comes when experts become overly 206 confident because they have been labelled as "experts". During this initial part of the interview, 207 the BN should be explained, indicating the uncertainty variables that will be elicited and explaining 208 how this process can be useful as regards the resolution of the overall problem. The second part of 209 this stage is concerned with structuring the variables: each quantity of interest that will be 210 quantified needs to be specified so that a measurement scale can be determined. Even if the 211 topology of the BN has already been defined, it is fundamental to review with the experts the 212 definitions of the variables and their states, in order to structure the uncertain quantities in an 
213 unambiguous and meaningful way, before starting with the encoding phase. Each variable must

214 have a clear definition that will be understood without any possibility of misunderstanding by the 215 expert. In addition, the states of every variable have to be determined in order to make 216 unambiguous the final estimation of the expert. It is common for a $\mathrm{BN}$ to represent the nodes with 217 discrete states: we suggest keeping them binary if possible, to minimize the number of variables 218 to quantify. Depending on the experience and mental models of the experts, it may be appropriate 219 to disaggregate the variable into more elemental variables. This can be very useful in the case of 220 the $\mathrm{BN}$, because each node might depend on several aspects and it can be easier for the experts to 221 evaluate these secondary probabilities. This technique also allows the analysts to combat the 222 motivational biases introduced at the beginning of this stage, i.e. the so-called management bias 223 and expert bias, and also some cognitive biases, e.g. the conjunctive bias, by increasing the level 224 of detail. The conjunctive bias is one of the biases associated to the anchoring heuristic (Tversky $225 \&$ Kahneman, 1974), which states that the overall probability is overestimated in conjunctive 226 problems and underestimated in disjunctive problems.

227 Stage 3: Encoding. This stage is concerned with encoding the expert's uncertainty on the 228 quantity of interest as a probability. Prior to eliciting these quantities training experts on probability 229 and providing relevant information for discussion should be conducted to minimize the presence 230 of potential biases (Tversky \& Kahneman, 1974) (Armstrong, et al., 1975). In particular, this can 231 address biases such as anchoring (Tversky \& Kahneman, 1974), i.e. when the evaluation is 232 conditioned by an initial assessment, and availability (Kahneman \& Tversky, 1973), i.e. when the 233 evaluation is based on the ease with which relevant instances come to mind. Probability training 234 should be provided to calibrate the experts: a brief review of basic probability concepts may be 235 helpful, along with some training questions which can help the experts to become familiar with 236 the elicitation process itself. Experts should be trained on problems relevant to the questions on 
237 which they will be providing judgement. When the training is completed, the encoding stage 238 commences. There are many available approaches to elicit probabilities, including direct 239 assessments of probabilities; for a review of methods we refer the Reader to (O'Hagan, et al., 2006). 240 A popular encoding procedure for distributions is the fractile method (Cooke 1991), where the expert 241 assesses the median value of their subjective probability distribution along with the $\left(25^{\text {th }}, 75^{\text {th }}\right)$ and the $242\left(5^{\text {th }}, 95^{\text {th }}\right)$ percentiles. Once the initial values have been elicited a parametric distribution can be 243 investigated and assessed for fit with the elicited values. The order in which these quantities are 244 elicited should start with the extreme values first and progress towards the central values, in order to 245 avoid the so-called central bias, i.e. the tendency to give an answer that is closer to the center of 246 opinions, and to not give an extreme answer. If the expert is uncomfortable with percentiles, 247 questions can be rephrased using qualitative bands, such as "highly likely" or "highly unlikely", 248 but the percentiles associated with these qualitative terms must be discussed and understood by 249 both expert and analysts. Alternately, graphical techniques (Chaloner, et al., 1993) may be useful 250 to improve the quality of the results. We recommend using the technique which makes the expert 251 more comfortable. In the case that there are a lot of probabilities to be elicited for the same node, 252 we suggest that the expert first ranks the factors from the most to the least influential and 253 subsequently quantifying the relationship, for instance following the swing weight method to 254 elicitation used with multi-attribute decision analysis (Belton \& Steward, 2001). Moreover, 255 sometimes it is not possible to elicit data for all the BN components, especially when it is composed 256 by a huge number of nodes or due to a limited time available. In this case, we recommend 257 identifying the quantities of interest that make the most significant contribution to the assessment 258 of the structure, for example through a sensitivity analysis (Li \& Mahadevan, 2018). Finally, 259 during the encoding phase, asking the same question in several ways can be a useful way of 
260 identifying potential inconsistencies with expert assessments. If this occur the expert should be

261 confronted and encouraged to reflect and respond on the assessments.

262 Stage 4: Verifying. This final stage starts by verifying the consistency of the elicited

263 probabilities. First of all, the analysts should verify that each expert has provided a reflection of

264 their true beliefs. Moreover, it is important to check for trends across the elicited probabilities to

265 determine if there are any indicators of anchoring bias or availability bias. If the results are not

266 satisfactory or biased, the previous stage should be repeated. In the case that the same conditional

267 probabilities have been elicited from different experts, the analysts should then develop an

268 aggregation technique to obtain one single final result; see (Quigley, et al., 2018) for a

269 performance-based approach or, if a consensus amongst experts is desired, see (Gosling, 2018) for

270 a behavioral based approach. Since the proposed methodology is based on discrete states, the final

271 stage of the SRI model, i.e. discretizing continuous probability distributions, is not needed. Once

272 each elicited probability has been verified and, if necessary, aggregated, the analysts should solve

273 the overall $\mathrm{BN}$ to achieve the final results. We suggest discussing with the experts also these final

274 outcomes in order to have a further validation of the developed process. After that, the interview

275 ends and the process can be considered concluded. 
Figure 2. Flowchart of the proposed elicitation process.

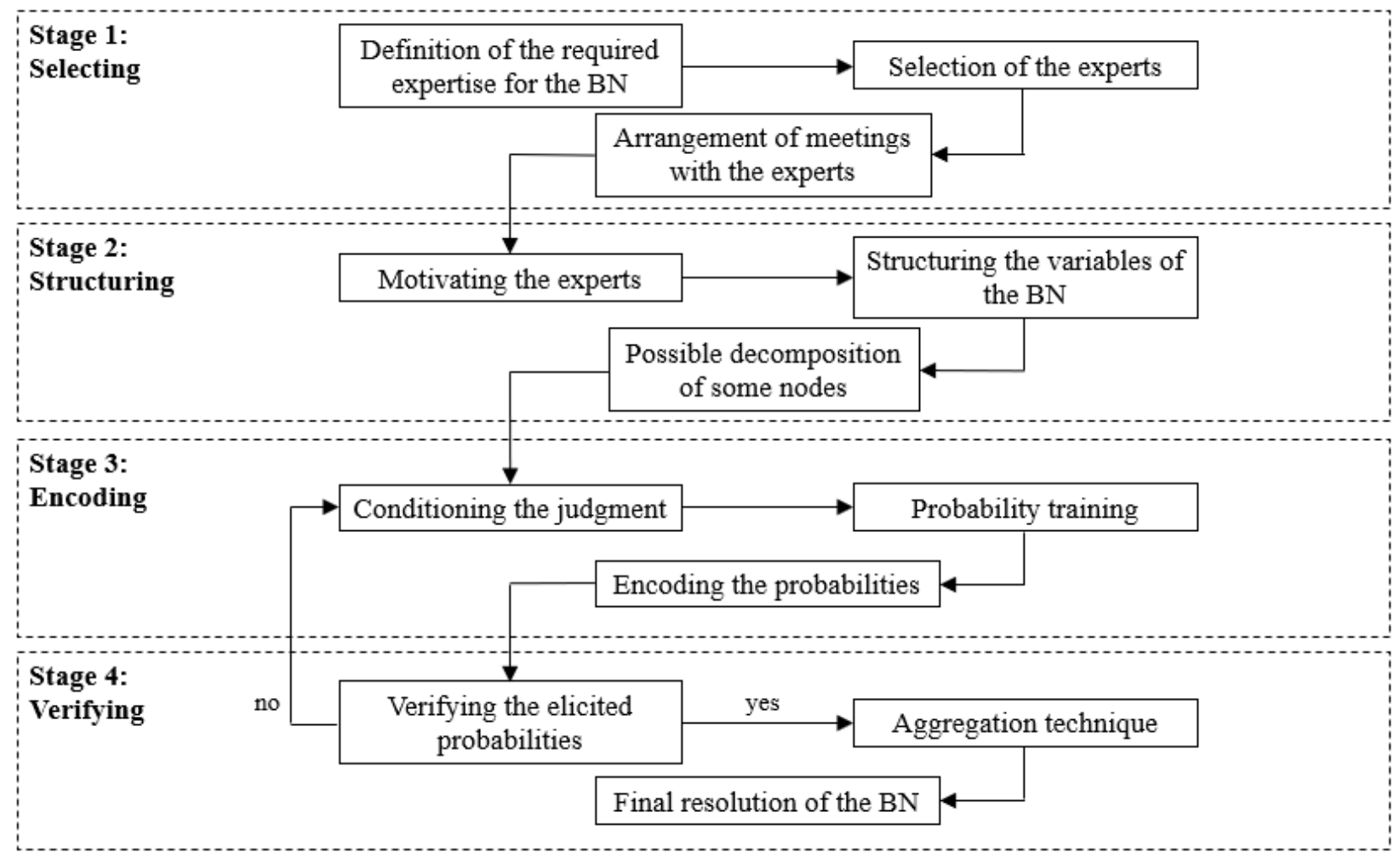

\section{The Mountain Chute Dam and GS case study}

The case study motivating our research is the Mountain Chute Dam and Generating Station

280 (GS), which is operated by Ontario Power Generation (OPG). Mountain Chute Dam and GS,

281 presented in Figure 3, is located in Greater Madawaska Township in Renfrew County (Ontario,

282 Canada): it has an electric power generation capacity of 170 megawatts of clean, renewable

283 electricity. It is situated on the Madawaska River, $64 \mathrm{~km}$ upstream from its confluence with the

284 Ottawa River, and it is in the upstream of four other hydroelectric facilities on the Madawaska

285 River: Barrett Chute GS, Calabogie GS, Stewartville GS and Arnprior GS. The construction started

286 in 1965 and was completed in December 1967. Three dams are located at the Mountain Chute GS:

287 one main concrete dam and two earthen block dams, i.e. the north block dam and the whitefish

288 draw dam. The main dam, shown in Figure 3(a), consists of the north and the south concrete gravity 
walls, the sluiceway and the headworks. It is $436 \mathrm{~m}$ long and $55 \mathrm{~m}$ above the rock foundations at

290 the deepest section; the elevation of the top of the concrete structure is $249.9 \mathrm{~m}$. The north block

291 dam, which is an embankment structure constructed across a shallow depression about $300 \mathrm{~m}$ north

292 east of the north abutment of the main dam north, is about $125 \mathrm{~m}$ long and has a maximum height

293 of $12 \mathrm{~m}$. Finally, the whitefish draw dam is a block dam preventing the reservoir from flowing out

294 via a side valley, it is located about $2.5 \mathrm{~km}$ north of the main dam, it is $204 \mathrm{~m}$ long and it has a

295 maximum height of $18 \mathrm{~m}$. More details about Mountain Chute GS and its case study are provided

296 in (El-Awady, et al., 2019) and (Verzobio, et al., 2019).

297 Figure 3. Mountain Chute Dam and GS: a) the main dam and the sluice gates; b) the downstream 298 of the dam; c) the upstream of the dam with the reservoir.

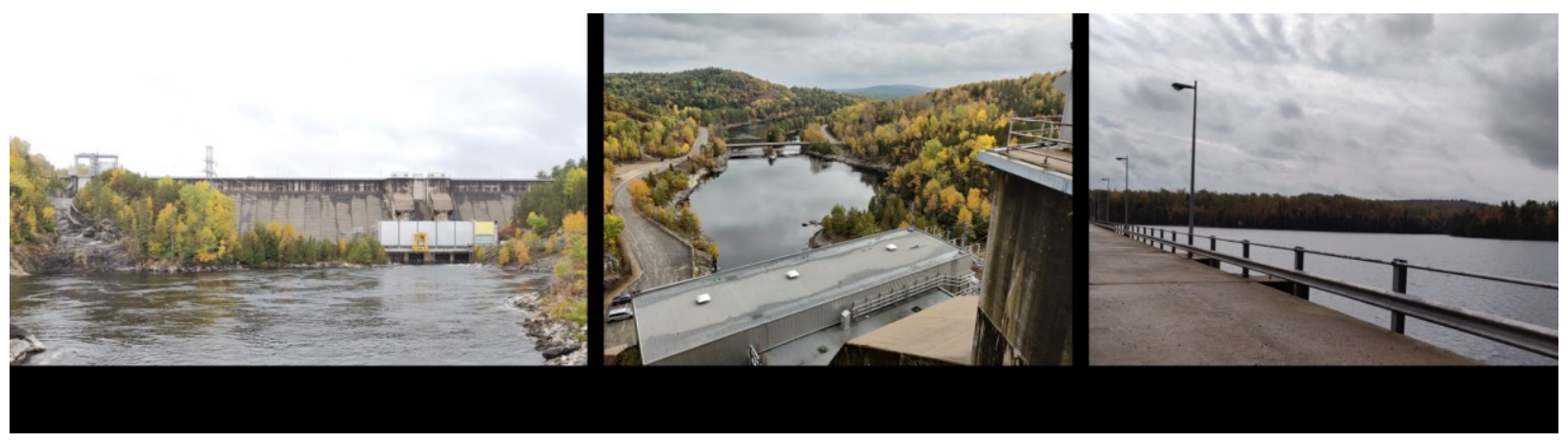

300 The main scope of this project is about the general safety of Mountain Chute, with the final aim

301 to estimate the probability of failure of the dams, intended as failure to perform at least one of the

302 required operations, according to the interrelated dam components. In the next subsections, we 303 describe the developed $\mathrm{BN}$ and successively the application of the proposed elicitation process, 304 which allows for improving the estimation of the failure probability of the dams, thanks to the 305 acquisition of valid and reliable data from expert knowledge. 


\subsection{Bayesian Network of Mountain Chute Dam and GS}

Mountain Chute station includes different kinds of system components. For the purpose of

308 analyzing the failure of this system, all system components should be defined, explained and

309 analyzed. Specifically, components such as rain precipitation, ice loading, earthquake and seismic

310 actions, water pressure, geology and rock type, flood severity, adequacy of discharge capacity,

311 sluice gate, drainage, vegetation control and other secondary components have to be considered.

312 A BN was constructed based on these components and based on the factors that can lead to the

313 failure of the dams, e.g. overtopping, seepage, sliding, stability issues and any operational failure,

314 such as problems related to the head gates or to the electromechanical equipment. The resultant

$315 \mathrm{BN}$ is presented in Figure 4.

316 Figure 4. Bayesian Network of Mountain Chute Dam and GS showing all the primary variables.

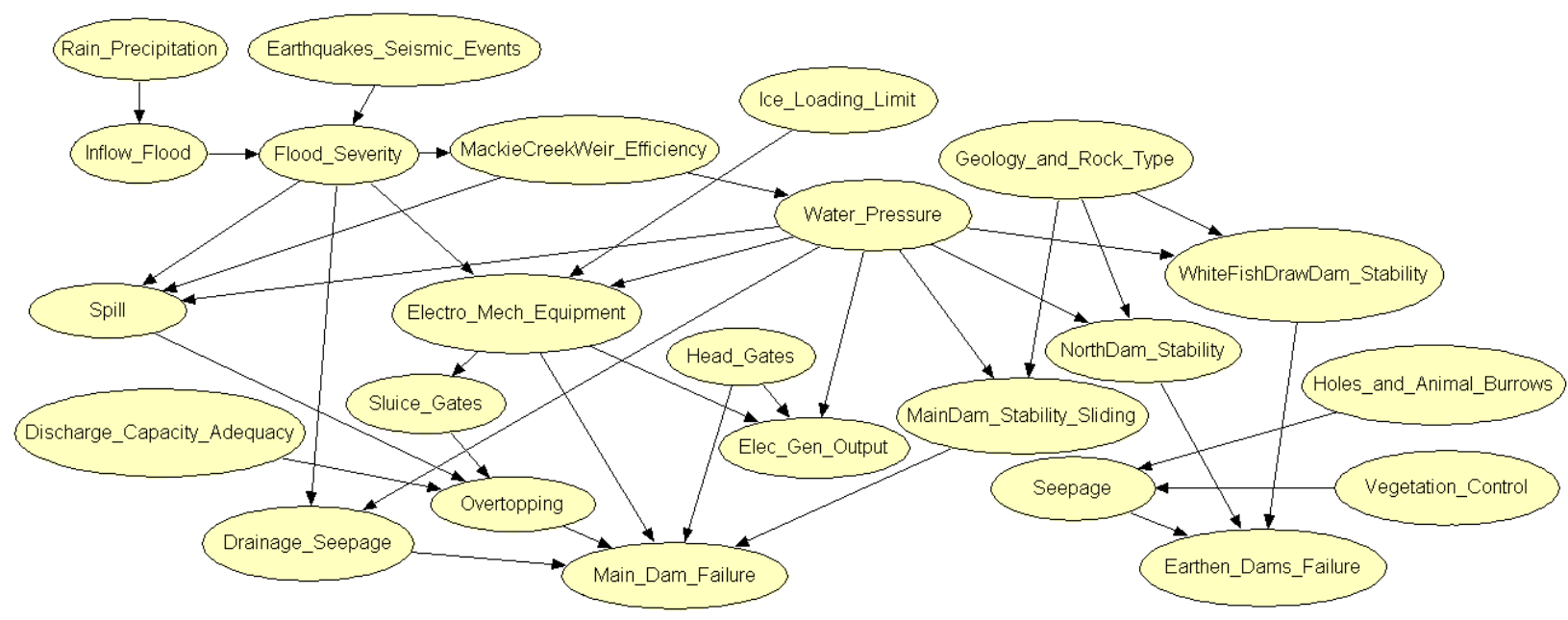

318 The main purpose of the developed BN, which is represented by 24 different nodes, i.e. the

319 yellow ovals in Figure 4, is predicting the probability of failure of the main dam from overtopping,

320 seepage, sliding or any operational failures. Moreover, it estimates the probability of failure of the

321 earthen block dams resulting from the threats of seepage and sliding. In the following, we analyze 
322 in detail the BN.

323 The basic events are rain precipitation, ice loading limits, earthquakes, geological and rock

324 stability, vegetation control and control of animal burrows. It can be seen from the BN that the

325 amount of rain affects the inflow to Mountain Chute dam; this inflow is considered a flood if it

326 exceeds a certain limit. If a flood takes place, it may be normal or severe. Flood severity is also

327 affected by seismic actions and earthquakes. The inflow rate and the severity level of the flood are

328 controlled by the Mackie Creek weir. Controlling the inflow is about preventing severe floods

329 from reaching the dam reservoir. The weir may be efficient or not, depending on the flood severity.

330 After passing the weir, the water in the reservoir, blocked by two earthen block dams and the main

331 concrete dam, is ready to be controlled by the dam head gates; this means that there is water

332 pressure behind the dams that may affect their stability. The geological and rock stability for the

333 structure of the three dams have been considered as it affects the sliding of the dam; sliding is one

334 of the causes of dam breach failure.

335 In addition, ice loading, water pressure and flood severity are connected to the 336 electromechanical equipment, including turbines; for instance, ice loading affects the failure of the

337 mechanical equipment and at the time of a severe flood and high-water pressure could result in

338 dam failure from maloperations of gates. As regards the electric power generation, the head gates

339 are opened to let the water flow through the penstock to generate electricity from hydropower

340 turbines. If the head gates fail to open, this is considered a failure of the main dam, especially if

341 the water pressure is high in the upstream side of the dam; this may affect the dam stability and

342 also the amount of power generated by the turbines.

343 Moreover, the flood severity, the weir efficiency in controlling the inflow to the reservoir and

344 the water pressure are all affecting the probability to have spill in the main dam; the spill is the

345 amount of water that exceeds the reservoir maximum capacity limit after considering various 
controlled outflows. This amount should be released from the upstream side to the downstream

347 side through the spillway (sluiceway) gates or an overtopping failure could take place. The amount

348 of water spill is also related to the capacity of sluiceway, which may not be adequate for that

349 amount of water to be discharged, and to the condition of the sluice gate, i.e. open or failed to open

350 due to electromechanical failure. If the water spill is not released from behind the main dam

351 because of the inadequate capacity of the sluiceway, or because the sluice gate fails to open, there

352 is an increasing probability, i.e. risk, of overtopping failure.

353 As concerns the main dam, severe floods with increased water pressure increases the possibility

354 to have seepage in the body of the main dam. If the seepage is not completely controlled and

355 monitored through a drain system which may include drain inspection tunnel, this would result in

356 an increasing risk that reduces the remaining lifetime of the dam. Finally, as regards the earthen

357 dams in Mountain Chute GS, seepage may take place because of uncontrolled vegetation and due

358 to animal burrows and holes in the vicinity of the dams. Seepage in the earthen block dams is then

359 an increasing risk for seepage piping and dam breach failure.

360 After the development of the topology of the BN with all its variables, the corresponding states

361 have been defined. It was clear that defining more than two states for every component of the BN

362 would have turned the system into a more complex network. On the other hand, more states would

363 have allowed to get more accurate results. Following the proposed methodology of the elicitation

364 process, due to the considerable number of nodes, it has been decided to keep the states of the

365 nodes binary, e.g. fail/no fail, safe/not safe, controlled/not controlled, efficient/not efficient. Table

3661 presents the defined states for each node. In addition, each state has been associated with a

367 detailed definition or a numerical value, so as to make them quantifiable. As an example, according

368 to the available data, the threshold according to which the rain precipitation passes from the state

369 low to the state high is when the rain depth reaches $60 \mathrm{~mm}$. 
Table 1. States of the BN variables.

\begin{tabular}{lcc}
\hline Variable & \multicolumn{2}{c}{ States } \\
\hline Rain precipitation & Low & High \\
Earthquakes seismic events & Normal & Severe \\
Ice loading limit & Safe & Not safe \\
Geology \& rock type & Stable & Unstable \\
Discharge capacity adequacy & Adequate & Not adequate \\
Head gates main dam & Open & Close/Fail to open \\
Holes and animal burrows & Controlled & Not controlled \\
Vegetation control & Controlled & Not controlled \\
Inflow flood & Low & High \\
Flood severity & Normal & Severe \\
Mackie Creek weir efficiency & Efficient & Not efficient \\
Water pressure & Normal & High \\
Spill & Yes & No \\
Electromechanical equipment main dam & Efficient & Not efficient \\
Sluice gates main dam & Open & Close/Fail to open \\
Electric generation output & Low & High \\
Overtopping & Yes & No \\
Drainage main dam seepage & Leakage & No leakage \\
Main dam stability sliding & Stable & Unstable \\
Main dam failure & Fail & No fail \\
North dam stability & Stable & Unstable \\
White fish drawn dam stability & Stable & Unstable \\
Seepage & Exist & Not exist \\
Earthen dams failure & Fail & No fail \\
\hline
\end{tabular}

371 Once the BN structure is completely defined, the conditional probability distributions were

372 determined based on logical inference and limited historical data; these probabilities are defined

373 to represent 100 years of operation for the Mountain Chute Dam and GS. Nevertheless, the

374 available data were not enough, and they did not allow to cover all the nodes of the BN. Then, it

375 was necessary to rely on expert judgment to provide subjective probabilities in order to populate

376 completely the model.

\section{$377 \quad 4.2 \quad$ Elicitation Process}

378 By following the methodology proposed in section 3, we implemented each stage of the process 
as follows.

380 Stage 1: Selecting. There were two analysts: one with knowledge in elicitation practice and 381 another with experience in the specific engineering area of failure analysis. After studying the 382 project and the defined model, we identified three areas of expertise from which we sought to elicit 383 expert judgment: structural stability expertise, environmental expertise and system design 384 expertise. While finding one expert per each area was desirable, due to availability constraints we 385 were given access to only one expert, who had a reasonable expertise in all the three areas: he was an engineer of the Ontario Power Generation who was responsible for monitoring the operations

387 of this specific GS. We were aware about the possible difficulty in finding available experts, but 388 managed to satisfy an essential coverage of expertise in all relevant area. A meeting was then 389 arranged at the site of the dam, in order to develop the interview. In preparation, the expert was 390 informed by email about the project and the specific aims of the interview.

391 Stage 2: Structuring. At the beginning of the interview the expert was motivated by explaining 392 the importance of the project, his fundamental rule and how the results will be used. Moreover, 393 the possible presence of motivational biases was investigated, especially the expert bias: it was 394 carefully pointed out to the expert that the goal is not to measure his personal expertise, but to 395 measure his knowledge about the events. Successively, we moved to the second part of this stage: 396 we reviewed the topology of the $\mathrm{BN}$ and the states of the variables together with the expert, to 397 ensure that there was no misunderstanding about their definition before moving to the encoding 398 phase. The expert therefore had the opportunity to review the topology of the BN but he decided 399 not to modify it, probably because we arrived at the meeting with a too refined model; he also 400 agreed with the proposed variables, refusing the possibility to disaggregate some nodes too. In 401 addition, we spent more time explaining meticulously to the expert the meaning of each variable 
402 and the corresponding states: after this discussion, and based on his opinions, we agreed to change

403 the definition as well as the threshold of some states.

404 Stage 3: Encoding. The encoding phase started by conditioning the expert's judgment in order

405 to avoid the possible presence of some cognitive biases. In particular, we focused mainly on the

406 anchoring, which is of particular concern with BNs given the large number of variables being

407 quantified: after the first assessment of the initial quantity of interest, the expert must avoid linking

408 the subsequent assessment with the previous one, as it would result in a biased adjustment.

409 Following this discussion, a probability training was carried out: we reviewed some probability

410 concepts and trained the expert with some specific questions similar to those that we would be

411 asked in the encoding phase, trying for instance to clarify the difference between a frequent event

412 and a very rare event. In addition, the probability scale illustrated in Figure 5 was introduced, that

413 we had established in order to help the expert during this stage of the process. This led to the

414 encoding phase, which was the most important and the longest, i.e. around 1 hour. It was developed

415 by asking questions in several ways, e.g. direct assessment of probabilities but also rephrasing the

416 questions using qualitative bands, to find potential inconsistencies in the answers and also to

417 reduce the influence of the explained biases. We chose these types of questions because we had

418 noticed that the expert was not completely comfortable using the percentiles. For example, we

419 asked the following questions: "What is the probability of a high inflow if the state of the rain

420 precipitation is low?"; "How frequently does it occur that the head gates of the main dam fail to

421 open?"; "How many days per year is it highly likely to have an inadequate capacity of sluiceway?"

422 During this phase it is important that the questions are very clear: for instance, we had to pay 423 attention to the reference time of each question in order to avoid misunderstanding with

424 interpreting the expert data, for example caused by the difference between the design time of a 425 dam and the real-life time of the dam. 
427 the results were satisfactory because the numerical outcomes seemed to coincide appropriately

428 with the true beliefs of the expert. Since we had the availability of just one expert, no aggregation

429 technique was necessary. Due to a limited time available the interview ended without the time to

430 solve the overall $\mathrm{BN}$ and to discuss the resulting outcomes, which would have been useful also as

431 an additional verification. In the end the interview lasted approximately two hours.

432 Figure 5. Probability scale used during the elicitation process.

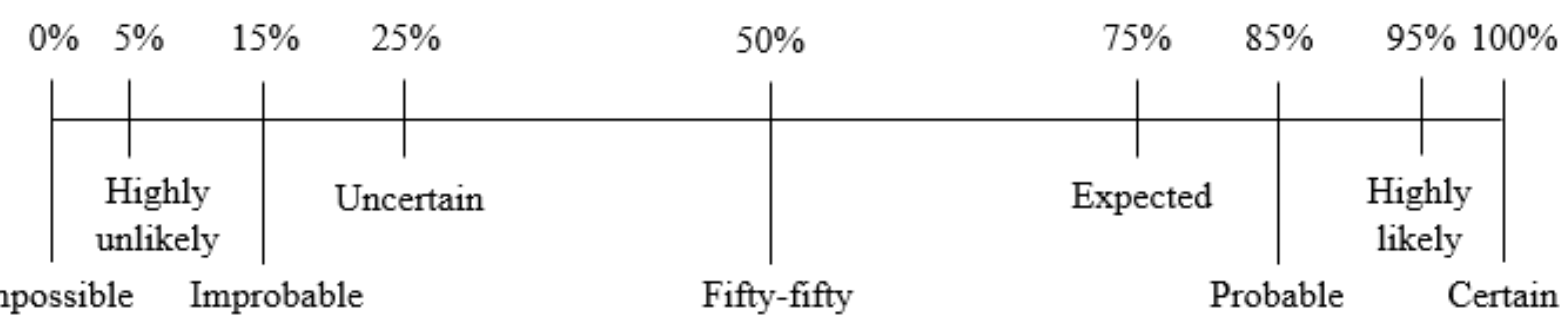

\section{$434 \quad 4.3 \quad$ Case study results}

435 In conclusion, after updating the probability distributions with data inferred from expert 436 engineering judgment as presented in the previous subsection, the overall BN was solved in order

437 to estimate the failure probabilities, which we remember are intended as failure to perform at least 438 one of the required operations. Figure 6 shows the results, achieved using the software Hugin: the 439 Bayesian inference results in a failure probability $\mathrm{p}_{\mathrm{F}}=0.0135$ for the main dam and $\mathrm{p}_{\mathrm{F}}=0.0133$

440 for the earthen block dams, both evaluated over the lifetime of the dams, i.e. 100 years. It is evident 441 that adding expert engineering judgments helps in reducing the uncertainties in the network, and 442 gives better estimates for the operation of the dam in comparison with those obtained using only 443 the limited available data and logical inference (El-Awady, et al., 2019). These final results about 444 the failure probability are satisfactory as they are close to those expected when considering these 
445 kind of systems design components: it provides approximately a failure of 1 in 10000 at any year

446 or equivalent to designing a dam for failure due to the so-called ten thousand years flood.

447 In addition, a BN is useful because explicates the cause-effect relationship, that is essential for

448 a better understanding of the dam safety. For instance, it is possible to understand the main

449 contributors to the failure of the main dam. Figure 7 shows the conditional probabilities of each

450 node given the main dam has failed. The most influential variables and the associated probabilities

451 are: seepage, i.e. 0.4551 leakage, electromechanical equipment, i.e. 0.2469 fail, sliding stability,

452 i.e. 0.2364 unstable, head gates, i.e. 0.2256 failed to open. On the other hand, overtopping has just

453 a probability of the 0.0795 .

454 Figure 6. The quantified BN of Mountain Chute Dam and GS (note that the numerical values are 455 percentage).

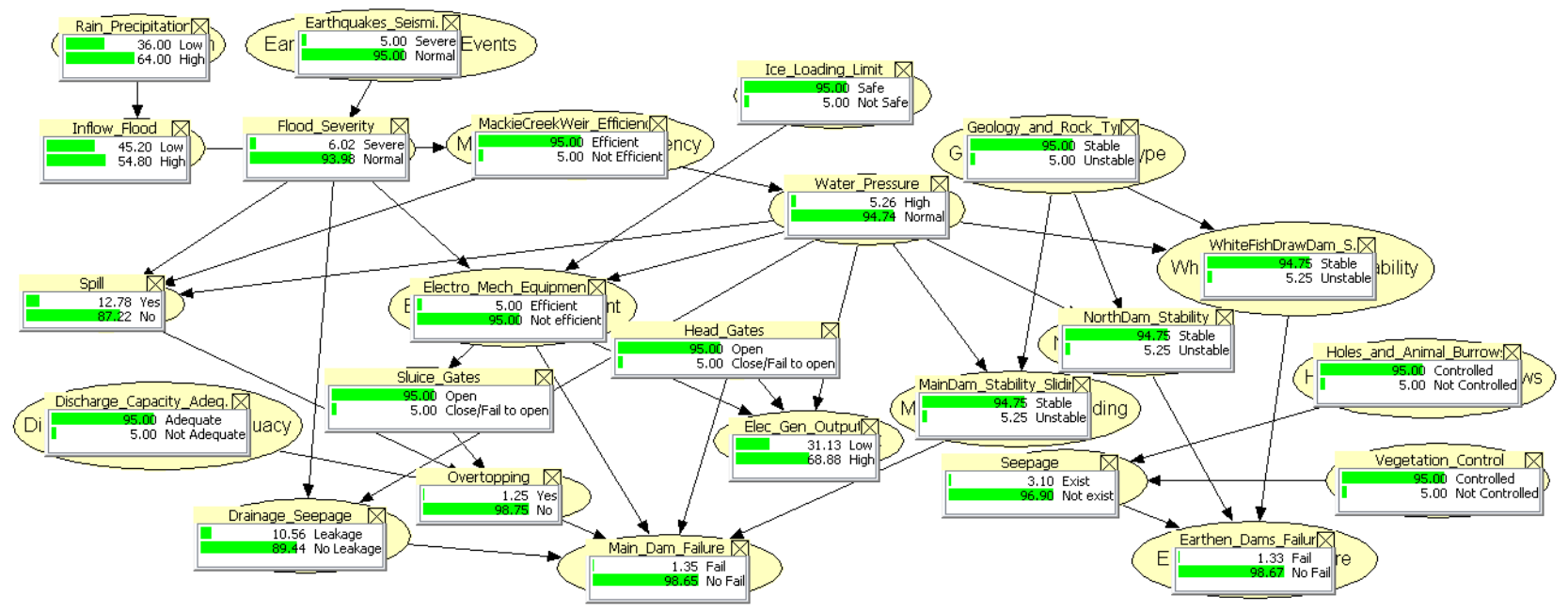


457 Figure 7. BN of Mountain Chute Dam and GS given the evidence that the main dam fails (note 458 that the numerical values are percentage).

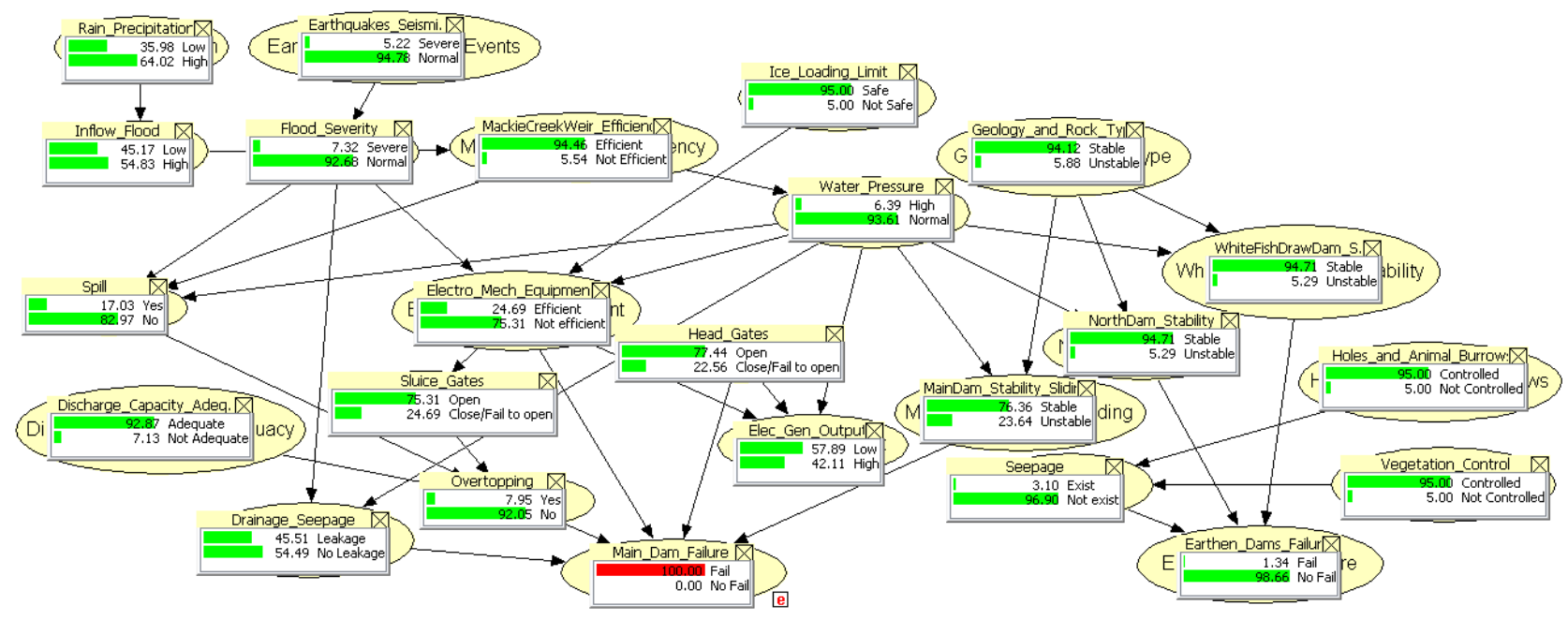

\subsection{Evaluation of the process}

After discussing the implementation of the planned elicitation process to this specific engineering case study and the consequent results, in this section we propose a critical discussion about the main steps of the process, based on what happened during its application, in order to understand how to improve the process and to give practical guidelines that can be used for similar 465 application in the future. working in a field where experts have narrow areas of expertise rather than generalists requires more experts to be involved in the elicitation to ensure sufficient coverage of the relevant issues. It is worthwhile reflecting on expertise that is desirable for the study or essential. In our case, even if we had the availability of only one expert, we managed to satisfy an essential coverage of expertise in all relevant area. For larger projects, expert 
$473 \quad$ Authority, 2014) (Bolger, 2018).

$474 \quad$ - As regards the number of analysts, the choice of two analysts with different competences

475 seemed appropriate: it is essential to have at least one facilitator with the expertise in the

476 elicitation practice that have to lead all the process, and at least another one with engineering

477 knowledge that have to make his contribution regarding the technical aspects of the specific

478 design project.

479 - The interview was conducted at the dam site: this choice has proved to be suitable because

480 it allowed us also to understand better some practical aspects of the dam operation. As regards the available time for the interview, we had scheduled a two-hour meeting but in the end we realised that it was not enough to properly complete all the planned elicitation process. During the scheduling phase we had probably underestimated some aspects of the interview that can lead to a delay, so we suggest detailed planning of the interview to identify an appropriate time.

486 - As concerns the structuring phase, we started with a very refined model, which can have some disadvantages, as it was evident that the expert did not propose many changes to the structure and agreed almost completely with our proposal; if the model had been less refined then the expert would have been more empowered to create a different model. Since this phase is fundamental in order to achieve accurate results during the encoding, we recommend involving the experts in the creation of the model and its variables.

492 - The training phase is fundamental to get accurate and reliable data from the experts. 493 Unfortunately, the time that we spent on training was too little, both because of the limited available time and because the expert did not seem too convinced about the importance of this phase. Consequently, we suggest adding a motivational phase at the beginning of this 
stage, i.e. encoding, in the same way as in the structuring stage, with the aim to explain to

497 the expert why it is necessary its development in order to calibrate him before encoding.

498 - There is a trade-off between the level of detail in a model and the time required to populate

499 with probabilities. The model structure needs to be flexible and adapt during the encoding,

500 as experts may not be comfortable expressing uncertainties on variables and require an

$501 \quad$ elaboration of the node.

502 - As concerns the encoding techniques, the choice to ask the questions with direct assessment

503 of probabilities and rephrasing the question using qualitative bands was made according to

504 the specific features of our expert: it was clear to us for instance that he was not comfortable

505 with the use of the percentiles. A good idea is then to prepare the questions in different ways

506 before the meeting, and to choose which ones to use only during the interview, so as to make

507 the expert as comfortable as possible.

508 - As regards the verifying stage, the limited available time did not let us to carry out it 509 completely. This is a problem that we have already highlighted and should be considered 510 properly during the scheduling phase. In particular, it would have been important to have 511 more time available in order to verify with the expert also the final resolution of the BN, 512 based on the elicited variables.

513 - Finally, during the implementation of all the stages we have paid close attention to the 514 possible presence of heuristics and biases, by following the appropriate actions suggested in 515 the methodology in order to minimize the risk of biased judgments. The achieved results 516 allow us to confirm the suitability of our four-stage elicitation process. 


\section{Conclusions}

BNs allow for analyzing complex systems like dams in order to develop a safety analysis based

519 on probabilistic estimates of failure. Due to the lack of data, in this paper we proposed a

520 methodology for an elicitation process aimed specifically at quantifying BNs, with the final goal

521 of collecting reliable data from engineering knowledge. The elicitation exercise we carried out for

522 this specific case study regarding the safety of the Mountain Chute Dam and GS, even if developed

523 in a simplified way, demonstrated the potential and the usefulness of the engineering expertise,

524 and allowed us to learn many lessons that are useful for improving the methodology, which we

525 intend to address in future for similar applications. In summary, we can conclude as follows:

526 - While the elicitation process has been applied in many fields, in civil engineering there is 527 little experience of applying formal elicitation processes to quantify models. This paper 528 demonstrates that engineering knowledge and experience can be very useful to solve 529 appropriately also this type of analysis.

$530 \quad-\quad$ It is undeniable that the elicitation requires a structured and facilitated process in order to 531 achieve accurate and reliable data, by avoiding the adverse impact of biases. However, there 532 is no perfect elicitation process: it has to be planned according to the particular context and 533 to the specific aims. Consequently, we proposed a detailed methodology for the precise aim 534 to quantify a BN.

535 - Our four-stage structured elicitation process works properly according to the results achieved 536 in the case study. However, this has been just our first experience in implementing an 537 elicitation process and instead, during the application, we have noticed some aspects that 538 need to be improved in order to make the process even more successful and reliable. 
539 - As regards to future work, we aim to improve this structured methodology based on what

540 we have learnt from this first application, and to apply it to other civil engineering structures,

$541 \quad$ e.g. bridges.

\section{Acknowledgements}

543 The authors would like to thank Ontario Power Generation, especially Tony Bennett and Andy

544 Zielinski, for letting us to work on such an important real-life case study, sharing all the required

545 material and allowing us to develop the interview with one of their engineers, whose availability 546 is greatly acknowledged.

\section{References}

548 Aguilera, P. A., Fernandez, A., Fernandez, R., Rumi, R., \& Salmeron, A. 2011. Bayesian networks

549 in environmental modeling. Environmental Modelling \& Software, 26(12): 1376-1388.

550 Ahmadi, A., Moridi, A. \& Han, D. 2015. Uncertainty Assessment in Environmental Risk through 551 Bayesian Networks. Journal of Environmental Informatics, 25(1): 46-59.

552 Armstrong, J. S., Denniston, W. B. \& Gordon, M. M. 1975. The use of decomposition principle in 553 making judgements. Organizational Behavior and Human Performance, 14(2): 257-263.

554 Astfalck, L., Cripps, E. L., Gosling, J. P., Hodkiewicz, M. R. \& Milne, I. A. 2018. Expert elicitation 555 of directional metocean parameters. Ocean Engineering, 161: 268-276.

556 Bayraktarli, Y., Ulfkjaer, J., Yazgan, U. \& Faber , M. 2005. On the application of Bayesian

557 probabilistic networks for earthquake risk management. Rome, Proc. 9th International $558 \quad$ Conference on Structural Safety and Reliability.

559 Belton, V. \& Steward, T. 2001. Multiple criteria decision analysis: an integrated approach. $560 \quad$ Dordrecht: Kluwer Academic Publishers. 
Ben Gal, I. 2007. Bayesian Networks. In: Ruggeri, Faltin \& Kennett, eds. Encyclopedia of $562 \quad$ Statistics in Quality \& Reliability. Chester: Wiley.

563 Bensi, M. T., Der Kiureghian, A. \& Straub, D. 2011. A Bayesian network methodology for 564 infrastructure seismic risk assessment and decision support. PEER Report 2011/02.

565 Bolger, F. 2018. The selection of Experts for (Probabilistic) Expert Knowledge Elicitation. In: $566 \quad$ Elicitation. Springer.

567 Briseno-Ramiro, R. A., Alcocer-Yamanaka, V. H., Pedrozo-Acuna, A., Brena-Naranjo, J. A. \& 568 Dominguez-Mora, R. 2019. Dam risk assessment using the event tree analysis and Bayesian 569 Networks. Panama City, Proc. 38th IAHR World Congress.

570 Bubniz, R. J., Apostolakis, G., Boore, D. M., Cluff, L. S., Coppersmith, K. J., Cornell, C. A. \& 571 Morris, P. A. 1998. Use of technical expert panels: Application to probabilistic seismic hazard $572 \quad$ analysis. Risk assessment, 18(4): 463-469.

573 Chaloner, K., Church, T., Lois, T. \& Mattis, J. 1993. Graphical Elicitation of a Prior Distribution 574 for a Clinical Trial. The Statistician, 42: 341-353.

575 Charniak, E. 1991. Bayesian networks without tears. AI magazine, 12(4): 50-63.

576 Christophersen, A., Deligne, N. I., Hanea, A. M., Chardot, L., Fournier, N. \& Aspinall, W. P. 2018. 577 Bayesian network modeling and expert elicitation for probabilistic eruption forecasting: pilot 578 study for Whakaari/White island, New Zeland. Frontiers in Earth Science, Volume 6.

579 Cooke, R. M. 1991. Experts in uncertainty. Oxford: Oxford University Press.

580 Dassanavake, S. M. \& Mousa, A. 2020. Probabilistic stability evaluation for wildlife-damaged 581 earth dams: a Bayesian approach. Georisk: Assessment and Management of Risk for $582 \quad$ Engineered Systems and Geohazards, 14(1): 41-55.

583 Dias, L. C., Morton, A. \& Quigley, J. 2018. Elicitation - The Science and Art of Strcturing $584 \quad$ Judgment. Springer. 
El-Awady, A., Ponnambalam, K., Bennett, T., Zielinsky, A. \& Verzobio, A. 2019. Bayesian Network Approach for Failure Prediction of Mountain Chute Dam and Generating Station. Ottawa, Ontario, Canada, ICOLD 87th annual meeting and symposium.

Eldosouky, A., Saad, W. \& Mandayam, N. 2017. Resilient Critical Infrastructure: Bayesian Network Analysis and Contract-Based Optimization. arXiv: 1709.00303.v1.

European Food Safety Authority. 2014. Guidance on expert knowledge elicitation in food and feed safety risk assessment. EFSA journal, 12(6).

Ferrell, W. 1985. Combining individual judgements. In: Wright G, editor. Behaviour decision making. New York: Plenum Press.

594 Ferrell, W. 1994. Discrete subjective probabilites and decision analysis: elicitation, calibration and 595 combination. In: Wright G, Ayton P, editors. Subjective probability. New York: Wiley.

596 Fischhoff, B. 1989. Eliciting knowledge for analytical representation. IEEE Trans Syst, Man 597 Cybernet, 19(3): 448-461.

598 Gang, W., Zhenyue, N., Jingjing, Q. \& Guan, L. 2016. Risk assessment method of earth rock-fill 599 dam break using multi-state and fuzzy basic events. Journal of Hydroelectric Engineering, $600 \quad 35(8): 95-104$.

601 Goodarzi, E., Mirzaei, M. \& Ziaei, M. 2012. Evaluation of dam overtopping risk based on 602 univariate and bivariate flood frequency analyses. Canadian Journal of Civil Engineering, $603 \quad 39(4): 374-387$.

604 Gosling, J. P. 2018. SHELF: the Sheffield elicitation framework. In: M. Q. Dias, ed. Elicitation: 605 the science and art of structuring judgment. New York: Springer.

606 Gret-Regamey, A. \& Straub, D. 2006. Spatially explicit avalanche risk assessment linking 607 Bayesian networks to a GIS. Natural Hazards and Earth Science, Copernicus Publications on 608 behalf of the European Geosciences Union, 6(6): 911-926. 
610 about reliability during design-lessons learnt from implementation. Quality and reliability 611 engineering international, 17(3): 169-179.

612 Jensen, F. V. \& Nielsen, T. D. 2007. Bayesian networks and decision graphs. 2nd ed. Berlin: $613 \quad$ Springer.

614 Jong, C. G. \& Leu, S. S. 2013. Bayesian-network-based hydro-power fault diagnosis 615 systemdevelopment by fault tree transformation. Journal of Marine Science and Technology, $61621(4): 367-379$.

617 Kahneman, D. \& Tversky, A. 1973. Availability: A Heuristic for Judging Frequency and 618 Probability. Cognitive Psychology, 5(2): 207-232.

619 Kelangath, S., Purnendu, K. D., Quigley, J. \& Hirdaris, S. E. 2011. Risk analysis of damaged ships 620 - a data-driven Bayesian approach. Ships and Offshore Structures, pp. 1-15.

621 Khakzad, N., Khan, F. \& Amyotte, P. 2011. Safety analysis in process facilities: Comparison of 622 fault tree and Bayesian network approaches. Reliability Engineering and System Safety, 96: $623 \quad 925-932$.

624 Langsetha, H. \& Portinaleb, L. 2007. Bayesian networks in reliability. Reliability Engineering and 625 System Safety, 92(1): 92-108.

626 Li, C. \& Mahadevan, S. 2018. Sensitivity analysis of a Bayesian Network. Journal of Risk and 627 Uncertainty in Engineering Systems, Volume 4.

628 Li, H., Li, J. \& Kang, F. 2011. Risk analysis of dam based on artificial bee colony algorithm with 629 fuzzy c-means clustering. Canadian Journal of Civil Engineering, 38(5): 483-492.

630 Ling, Y. \& Mahadevan, S. 2012. Integration of Structural Health Monitoring and Fatigue Damage 631 Prognosis. Mechanical Systems and Signal Processing, 28: 89-104. 
632 Liu, Z. Q. \& Nadim, F. 2013. Publications on Quantitative risk assessment for eartquake-triggered 633 landslides using Bayesian network. Paris, Proc. 18th International Conference on Soil 634 Mechanics and Geotechnical Engineering.

635 Liu, Z. Q., Nadim, F., Eidsvig, U. K. \& Lacasse, S. 2017. Reassessment of Dam Safety Using 636 Bayesian Network.. Geo-Risk, pp. 168-177.

637 Maroni, A., Tubaldi, E., Val, D., McDonald, H., Lothian S., Riches, O. \& Zonta, D. 2019. A 638 decision support systemfor scour management of road and railway bridges based on Bayesian 639 networks. Stanford, Proc. 12th International Workshop on Structural Health Monitoring.

640 Medina-Cetina, Z. \& Nadim, F. 2008. Stochastic design of an early warning system. Georisk: 641 Assessment and Management of Risk for Engineerign Systems and Geohazards, 2(4): 223-236.

642 Merkhofer, M. 1987. Quantifying judgemental uncertainty: methodology, experiences, and 643 insights. IEEE Trans Syst, Man Cybernet, 17(5): 741-752.

644 Meyer, M. \& Booker, J. 1991. Eliciting and analyzing expert judgment - a practical guide. New $645 \quad$ York: Academic Press.

646 Miroslaw-Swiatek, D., Kemblowski, M. \& Jankowski, W. 2012. Application of the Bayesian 647 Belief Nets in dam safety monitoring. Annals of Warsaw University of Life Sciences - SGGW. $648 \quad$ Land reclamation, 44(1): 25-33.

649 Morgan, M. G., Small, M. J. \& Henrion, M. 1990. Uncertainty: a guide to dealing with uncertainty 650 in quantitative risk and policy analysis. New York: Cambridge University Press.

651 Norrington, L., Quigley, J., Russell, A. \& Van der Meer, R. 2008. Modelling the reliability of 652 search and rescue operations with Bayesian Belief Networks. Reliability Engineering and $653 \quad$ System Safety, 93(7): 940-949.

654 O'Hagan, A., Buck, C., Daneshkhah, A., Eiser, R., Garthwaite, P., Jenkinson, D., Oakley, J. \& 655 Rakov, T. 2006. Uncertainty Judgments: Eliciting Experts' Probabilities. Wiley. 
Peng, M. \& Zhang, L. M. 2013. Dynamic decision making for dam-break emergency management

657 - Part 1: Theoretical framework. Natural Hazards \& Earth System Sciences, 13: 425-437.

658 Peter, C., De Lange, W., Musango, J. K., April, K. \& Potgieter, A. 2009. Applying Bayesian

659 modelling to assess climate change effects on biofuel production. Climate research, 40: 240-

660260.

661 Quigley, J., Colson, A., Aspinall, W. \& Cooke, R. 2018. Elicitation in the Classical Model. In:

662 Dias, Morton \& Quigley, eds. Elicitation: The Science and Art of Structuring Judgement. 663 Springer.

664 Quigley, J. \& Walls, L. 2020. Characteristics of a Process for Subjective Probability Elicitation.

665 In: French, Bedford, Nane \& Hanea, eds. Expert judgment in Risk and Decision Analysis.

666 Springer International Publishing.

667 Rizak, S. \& Hrudey, S. E. 2005. Interdisciplinary comparison of expert risk beliefs. Journal of 668 Environmental Engineering Science, 4(3): 173-185.

669 Sankararaman, S., Ling, Y. \& Mahadevan, S. 2011. Uncertainty Quantification and Model 670 Validation of Fatigue Crack Growth Prediction. Engineering Fracture Mechanics, 78(7): 1487$671 \quad 1504$.

672 Sigurdsson, J. H., Walls, L. A. \& Quigley, J. 2001. Bayesian belief nets for managing expert 673 judgment and modelling reliability. Quality and reliability engineering international, 17: 181$674 \quad 190$.

675 Smith, M. 2006. Dam Risk Analysis Using Bayesian Networks. Lillehammer, Norway, Proc. 676 Engineering Conferences International.

677 Spetzler, C. \& Stael Von Holstein, C. A. 1985. Probability Encoding in Decision Analysis. TIMS, $678 \quad 22: 340-358$. 
679 Su, H., Wen, Z., Xiaoran, S. \& Yang, M. 2015. Time-varying identification model for dam 680 behavior considering structural reinforcement. Structural Safety, 57: 1-7.

681 Tversky, A. \& Kahneman, D. 1974. Judgment under Uncertainty: Heuristics and Biases. Science, $682 \quad$ New Series, pp. 1124-1131.

683 Verzobio, A., El-Awady, A., Ponnambalam, K., Quigley, J. \& Zonta, D. 2019. Elicitation Process 684 to Populate Bayesian Networks with Application to Dam Safety. Nanjing, China, 8th 685 International Conference on Water Resources and Environmental Research (ICWRER).

686 Vick, S. G. 2002. Degrees of Belief: Subjective Probability and Engineering Judgment. Reston, 687 VA: ASCE Press.

688 Xu, Y., Zhang, L. M. \& Jia, J. S. 2011. Diagnosis of embankment dam distresses using Bayesian 689 networks. Part II: Diagnosis of a specific distressed dam. Canadian geotechnical journal, pp. $690 \quad 1645-1657$.

691 Yanmaz, A. M. \& Gunindi, M. E. 2008. Assessment of overtopping reliability and benefits of a 692 flood detention dam. Canadian Journal of Civil Engineering, 35(10): 1177-1182.

693 Zerrouki, H. \& Tamrabet, A. 2015a. Fault tree and Bayesian Network in safety analysis: 694 Comparative study. Djelfa, Algeria, Proc. International Conference on Applied Automation and 695 Industrial Diagnostics .

696 Zerruki, H. \& Tamrabet, A. 2015b. Mapping Fault Tree into Bayesian Network in safety analysis 697 of process system. Boumerdes, Proc. 4th International Conference on Electrical Engineering. 698 Zhang, L. M., Xu, Y., Jia, J. S. \& Zhao, C. 2011. Diagnosis of embankment dam distresses using 699 Bayesian networks. Part I: Global-level characteristics based on a dam distress database. $700 \quad$ Canadian geotechnical journal, pp. 1630-1644. 
704 Table 1. States of the BN variables.

\begin{tabular}{lcc}
\hline Variable & & States \\
\hline Rain precipitation & Low & High \\
Earthquakes seismic events & Normal & Severe \\
Ice loading limit & Safe & Not safe \\
Geology \& rock type & Stable & Unstable \\
Discharge capacity adequacy & Adequate & Not adequate \\
Head gates main dam & Open & Close/Fail to open \\
Holes and animal burrows & Controlled & Not controlled \\
Vegetation control & Controlled & Not controlled \\
Inflow flood & Low & High \\
Flood severity & Normal & Severe \\
Mackie Creek weir efficiency & Efficient & Not efficient \\
Water pressure & Normal & High \\
Spill & Yes & No \\
Electromechanical equipment main dam & Efficient & Not efficient \\
Sluice gates main dam & Open & Close/Fail to open \\
Electric generation output & Low & High \\
Overtopping & Yes & No \\
Drainage main dam seepage & Leakage & No leakage \\
Main dam stability sliding & Stable & Unstable \\
Main dam failure & Fail & No fail \\
North dam stability & Stable & Unstable \\
White fish drawn dam stability & Stable & Unstable \\
Seepage & Exist & Not exist \\
Earthen dams failure & Fail & No fail \\
\hline
\end{tabular}


707 Figure 1. An example of BN with three variables.

708 Figure 2. Flowchart of the proposed elicitation process.

709 Figure 3. Mountain Chute Dam and GS: a) the main dam and the sluice gates; b) the downstream 710 of the dam; c) the upstream of the dam with the reservoir.

711 Figure 4. Bayesian Network of Mountain Chute Dam and GS showing all the primary variables.

712 Figure 5. Probability scale used during the elicitation process.

713 Figure 6. The quantified BN of Mountain Chute Dam and GS (note that the numerical values are 714 percentage).

715 Figure 7. BN of Mountain Chute Dam and GS given the evidence that the main dam fails (note 716 that the numerical values are percentage). 